A continuum plasticity model that accounts for hardening and size effects in thin films

This article has been downloaded from IOPscience. Please scroll down to see the full text article.

2010 Modelling Simul. Mater. Sci. Eng. 18045012

(http://iopscience.iop.org/0965-0393/18/4/045012)

View the table of contents for this issue, or go to the journal homepage for more

Download details:

IP Address: 128.210.126.199

The article was downloaded on 24/10/2010 at 05:46

Please note that terms and conditions apply. 


\title{
A continuum plasticity model that accounts for hardening and size effects in thin films
}

\author{
Abigail Hunter, H Kavuri and Marisol Koslowski \\ School of Mechanical Engineering, Purdue University, West Lafayette, IN 47906, USA
}

Received 21 September 2009, in final form 24 March 2010

Published 16 April 2010

Online at stacks.iop.org/MSMSE/18/045012

\begin{abstract}
We conducted three-dimensional finite element simulations of the mechanical response of passivated single crystal copper thin films with a continuum crystal plasticity model. The model introduces the formation of high density dislocation layers close to the substrate and passivation interfaces obtained from dislocation dynamics simulations. These dislocation structures are responsible for an increase in strain hardening as the film thickness decreases. The model predicts an increase in strain hardening as the film thickness decreases in agreement with experimental observation in films with thickness in the range 0.2 to $2 \mu \mathrm{m}$.
\end{abstract}

(Some figures in this article are in colour only in the electronic version)

\section{Introduction}

The continuing shrinking of dimensions and increased complexity in micrometer size devices, such as microelectronic interconnects and micro-electro-mechanical systems (MEMS), results in the emergence of new mechanical problems during manufacturing and operation. Even though plastic deformation is not a key failure mechanism in these devices, crystalline structure, grain boundaries and interfaces play an important role by affecting the dislocation and vacancy concentrations and affecting the response and reliability of these devices.

The plastic response of metallic thin films shows size dependence when the film thickness or another characteristic length such as the grain size approaches the micrometer or submicrometer range. This size dependence is given by an increase in the yield stress as the characteristic size decreases. This relation, known as Hall-Petch relation [1,2], is well established experimentally in polycrystalline materials with sub-micrometer and micrometer grain size. Experiments conducted with thin films with passivated interfaces show that in crystalline materials the value of the yield stress increases as the film thickness decreases following a relation proportional to $h^{-n}$, where $h$ is the film thickness [3-6] and the exponent $n$ ranges from 0.1 to 1 . A wide range in the value of the exponent is observed due to the fact that the yield stress also is influenced by the microstructure, grain size and substrate and passivation 
material and thickness and these effects cannot in general be isolated. The dependence of this hardening relation on the thin film thickness seems to be stronger than that on the grain size in $\mathrm{Cu}$ and $\mathrm{Al}$, where the grain size is on the same order as the film thickness [5-7]. On the other hand, in Ni thin films the size effects due to grain size are evident [8]. A more detailed characterization seems to be needed to decouple the effects of grain size and film thickness as usually both are related.

Strengthening of thin film materials has been the subject of intensive research over many decades [9-14]. The scaling behavior of the resolved flow shear stress, $\tau_{y}$, can be written in general as

$$
\tau_{y}=\tau_{0}+\alpha \mu \frac{b}{h^{n}},
$$

where $\tau_{0}$ is the resolved flow shear stress of the bulk material, $\mu$ is the shear modulus, $b$ is the Burgers vector, $\alpha$ is the scaling parameter. Freund [10] and Nix [11] found an exponent $n=1$ for the above relation based on studies with a single dislocation moving between two hard interfaces. In this approximation this single dislocation advances under an external stress leaving a dislocation line along the interface. Recent simulations with a dislocation phasefield model found the same exponent for the yield stress [14, 15]. In these simulations several dislocations are considered and a closed form solution of the form of equation (1.1) is obtained for steady state. This solution shows that dislocations form pile ups made of dislocation segments against the hard interfaces in agreement with the Freund model [10] and that these dislocation pile ups are responsible for the strengthening of thin films. Similar dislocation structures are observed in passivated thin film experiments $[4,16]$ and dislocation dynamics simulations $[12,15]$.

On the other hand film thickness also affects strain hardening in thin films. Experiments show that in thin films with over $100 \mathrm{~nm}$ thickness strain hardening increases as the film decreases $[6,4,17,18]$. While films with thickness below $100 \mathrm{~nm}$ show very reduced hardening. Gruber et al [6] calculated the difference in stress, $\Delta \sigma$, for two different strain values $(0.5 \%$ and $0.1 \%)$ as a function of the film thickness. In their results strain hardening increases as the film thickness decreases following a power law of the form

$$
\Delta \sigma \approx \alpha_{d} h^{-n_{d}}
$$

with $n_{d} \approx 0.6$.

In general, continuum plasticity models follow the evolution of the dislocation density $[19,20]$. These models were originally developed for bulk materials and do not take into account boundary conditions for the dislocation density at interfaces such as grain boundaries and passivation that are responsible for size effects when the domain size approaches the characteristic size of the microstructure. Several continuum plasticity approaches were recently developed to account for these effects. These models in general add terms proportional to the gradient of the plastic deformation and a length scale parameter to account for size effects [21-25].

It is clear that size effects in the yield stress can be predicted only if individual dislocations and their interactions are taken into account $[12,15]$, while strain hardening may be the result of dislocation accumulation in reduced volumes and can be described in terms of the dislocation density. Here, we will concentrate on the dependence of strain hardening in film thickness. To this end we couple the evolution of the dislocation density obtained from direct simulations of dislocations into a continuum plasticity model.

We simulate a $\mathrm{Cu}$ single crystal thin film constrained by a substrate and passivation under thermal cycling. The thickness of the thin film is between 0.2 and $2 \mu \mathrm{m}$ and therefore size effects due to grain size are not included. The model is able to predict hardening for decreasing 
film thickness in agreement with experimental observation. The evolution of the dislocation density shows a high density close to interfaces.

\section{Single crystal plasticity}

In this section we introduce the general formulation for the continuum single crystal plasticity theory used in the simulations. The kinematics is based on the model developed by Cuitino and Ortiz [20]. Following this model we consider that the deformation gradient, $\boldsymbol{F}$, may be assumed to be decomposed multiplicatively into an elastic part, $\boldsymbol{F}^{\boldsymbol{e}}$, describing the lattice deformation and a plastic part, $\boldsymbol{F}^{p}$, providing the cumulative effect of the dislocation motion:

$$
\boldsymbol{F}=\boldsymbol{F}^{e} \boldsymbol{F}^{p} .
$$

Considering that the material undergoing plastic deformation is face center cubic (fcc), the plastic part of the deformation gradient can be written as the sum of the slip on each of the 12 systems of a fcc crystal, following [19]

$$
\boldsymbol{F}^{p}=\boldsymbol{I}+\sum_{\alpha} \gamma^{\alpha} \boldsymbol{s}^{\alpha} \otimes \boldsymbol{m}^{\alpha}
$$

where $\gamma$ is the shear strain on the glide plane, $s^{\alpha}$ is the slip direction and $\boldsymbol{m}^{\alpha}$ is normal to the slip plane $\alpha$ [19]. The plastic flow rule results

$$
\boldsymbol{L}^{p}=\sum_{\alpha} \gamma^{\alpha} \boldsymbol{s}^{\alpha} \otimes \boldsymbol{m}^{\alpha}
$$

where the sum is over all the slip systems and $\boldsymbol{L}^{p}=\boldsymbol{F}^{p} \boldsymbol{F}^{p-1}$ is the plastic velocity gradient and $\gamma^{\alpha}$ is the shear rate on system $\alpha$. Following [20] we assume that the rate of shear deformation in the slip plane $\alpha$ is given by a relation of the form

$$
\dot{\gamma}^{\alpha}= \begin{cases}\dot{\gamma}_{0}^{\alpha}\left(\tau^{\alpha} / \tau_{y}^{\alpha}\right)^{1 / m} & \text { if } \tau>\tau_{y}, \\ 0 & \text { otherwise, }\end{cases}
$$

where $\gamma_{0}^{\alpha}$ is a reference strain rate , $\tau^{\alpha}$ is the resolved shear stress in the slip plane $\alpha, \tau_{y}^{\alpha}$ is the flow stress in the slip system $\alpha$ and $m$ is a hardening exponent set to 0.01 in the current simulations. The evolution of the flow stress is governed by the hardening law:

$$
\dot{\tau_{y}^{\alpha}}=\sum_{\beta} h^{\alpha \beta} \gamma^{\beta}
$$

where $h^{\alpha \beta}$ are hardening moduli coefficients; these hardening coefficients are not constant but depend on the state of deformation and the local dislocation density. Following [20] we assume that the hardening coefficients are of the form

$$
h^{\alpha \beta}=\frac{a \mu n^{\alpha}}{\rho^{\alpha}}\left(\frac{\tau_{y}^{\alpha}}{\tau_{o}^{\alpha}}\right)^{3}\left(\cosh \left(\frac{\tau_{0}^{\alpha}}{\tau_{y}^{\alpha}}\right)^{2}-1\right) \delta_{\alpha \beta},
$$

where $\tau_{0}^{\alpha}$ is a characteristic flow stress that follows Taylor's hardening law $\tau_{0}^{\alpha}=a \mu b \sqrt{n^{\alpha}}$, $a$ is a constant coefficient set to $0.3, b$ is the Burgers vector, $\mu$ is the shear modulus and $n^{\alpha}$ is the number of forest dislocations piercing the slip plane $\alpha$ that depends on the dislocation densities, $\rho$, on other slip systems, following

$$
n^{\alpha}=\sum_{\beta} a^{\alpha \beta} \rho^{\beta} \text {. }
$$


Values of the interaction matrix $a^{\alpha \beta}$ were determined experimentally by Franciosi and Zaoui [26] for fcc materials and those values were used in the current simulations.

In general the constitutive equation for the dislocation density evolution in continuum models follows a phenomenological equation that accounts for dislocation multiplication and annihilation. The most general expression is of the form

$$
\rho^{\alpha}=\rho^{\alpha}\left(\rho_{\mathrm{sat}}, \rho_{0}, \gamma^{\alpha}\right),
$$

where $\rho_{0}$ and $\rho_{\text {sat }}$ are the initial and saturation density, respectively. The evolution of the dislocation density on each slip plane and its dependence on the plastic slip $\gamma^{\alpha}$ determines the rate of hardening in the present model as follows. During the initial stage of deformation glides occur predominantly in the slip system with the biggest resolved shear stress. The number of obstacles in the inactive slip systems increases due to dislocation multiplication in the active system. This has the effect of a rising the value of the critical stresses $\tau_{0}$ in the other systems. This effect, called latent hardening, is observed in experiments. On the other hand, the effect of self-hardening is clear from equations (2.5) and (2.6) and Taylor hardening law.

In the following section we will introduce an evolution equation for the dislocation density derived from a phase-field dislocation theory that accounts for dislocation interactions with hard interfaces and integrates size effects into the single crystal plasticity model described in this section.

\section{Dislocation density evolution in metallic thin films}

In bulk materials the strength is determined by the resistance to dislocation motion due to dislocation dislocation interactions and dislocations interacting with impurities, second phase particles and grain boundaries. As the grain size decreases the ratio between the grain boundary and the volume of the grain increases and the interaction of dislocations with grain boundaries plays a prominent role in plastic deformation. In thin film materials film thickness is of the order of the grain size and dislocation motion is also constrained by the substrate and the passivation layer. This confinement in the dislocation motion is responsible for an increase in the yield stress as the film thickness decreases.

The dislocation density is the microscopic quantity that we will use to make the connection to the macroscopic model in the current theory. To this end, we will describe the evolution of the dislocation density following the phase-field theory of dislocations developed by Koslowski et al $[14,15,27]$. We will describe the evolution of the dislocation density in an individual slip plane where dislocation motion is constrained by impenetrable interfaces as shown in figure 1 .

In the phase-field dislocation model dislocations are represented by a scalar field, $\zeta^{\alpha}(\boldsymbol{x})$, for each slip plane $\alpha$. This scalar field represents the amount of slip on units of the Burgers vector on the slip plane. Under these assumptions the evolution of the dislocation field in a single slip system is described by a time dependent Ginzburg-Landau equation [14]:

$$
\frac{\partial \zeta^{\alpha}(\boldsymbol{x})}{\partial t}=-L\left(\kappa\left[\zeta^{\alpha}(\boldsymbol{x})\right]-\tau^{\alpha}+\eta^{\alpha}(\boldsymbol{x})\right),
$$

where $L$ is a kinetic coefficient, the first term on the right represents the dislocation interactions, $\tau^{\alpha}$ is the resolved shear stress in the slip plane $\alpha, \eta^{\alpha}(\boldsymbol{x})$ represents the interaction with obstacles. In the first term $\kappa\left[\zeta^{\alpha}(\boldsymbol{x})\right]$ is a non-local kernel that has the following form [27, 28]:

$\kappa\left[\zeta^{\alpha}(\boldsymbol{x})\right]=\int \frac{1}{2 \pi(1-v)|\boldsymbol{x}-\boldsymbol{y}|^{3}}\left(v+1-3 v \frac{x_{1}^{2}-y_{1}^{2}}{|\boldsymbol{x}-\boldsymbol{y}|^{2}}\right)\left(\zeta^{\alpha}(\boldsymbol{x})-\zeta^{\alpha}(\boldsymbol{y})\right) \mathrm{d} y$.

We assume that the strain rate is slow enough such that the (3.1) reaches equilibrium at each time step. Therefore, at each time step the dislocation structure is the solution to the 


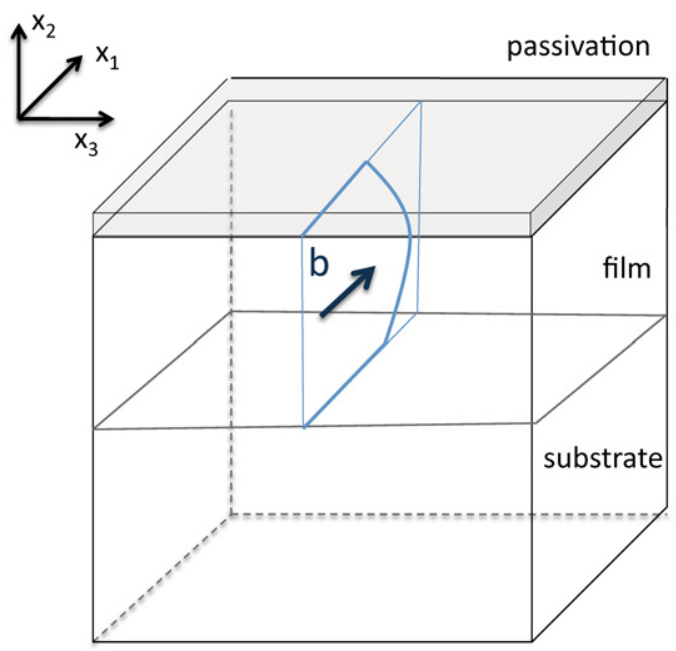

Figure 1. Geometry of a dislocation moving in a single slip plane in a thin film.

steady state equation

$$
\kappa\left[\zeta^{\alpha}(\boldsymbol{x})\right]-\tau^{\alpha}+\eta^{\alpha}(\boldsymbol{x})=0 .
$$

The solution to this equation can be found in closed form in the presence of point obstacles [27] or next to impenetrable interfaces $[14,15,29]$ and results

$$
\zeta^{\alpha}\left(x_{2}\right)=G \sqrt{x_{2} / h-x_{2}^{2} / h^{2}} \quad \text { with } \quad G=\left(\tau^{\alpha}-\tau_{\eta}^{\alpha}\right) \frac{2}{\mu(b / h)},
$$

where $x_{2}=0$ and $x_{2}=h$ are the interfaces with the following boundary conditions

$$
\zeta\left(x_{2}\right)=0 \quad \text { on } \quad x_{2}=0, h,
$$

where $\tau_{\eta}^{\alpha}$ accounts for the resistance of the obstacles and represents an average of $\eta^{\alpha}(\boldsymbol{x})$ in (3.3). The boundary conditions in (3.5) state that dislocation cannot penetrate the interface between the thin film and substrate and passivation.

The connection with the continuous model described in section 2 can be achieved by finding an expression that related the dislocation density to the plastic strain. Following [27] the dislocation density can be obtained directly as the gradient of the phase field as

$$
\rho^{\alpha}(\boldsymbol{x})=\frac{1}{w}\left|\nabla \zeta^{\alpha}(\boldsymbol{x})\right|+\rho_{0},
$$

where $w$ is the slip plane distance, which is assumed to be known and $\rho_{0}$ is the initial dislocation density, i.e. the dislocation density at zero applied stress. On the other hand, we define the average plastic slip as the average of the phase field $\zeta^{\alpha}(x)$ over the film thickness, $h$, for each slip system $\alpha$ as

$$
\bar{\gamma}^{\alpha}=\frac{b}{h w} \int_{0}^{h} G \sqrt{x_{2} / h-x_{2}^{2} / h}=\frac{h \pi}{4 w \mu}\left(\tau^{\alpha}-\tau_{\eta}^{\alpha}\right) .
$$

Replacing (3.4) and (3.7) in (3.6) we obtain a relation for the dislocation density profile in a thin film for an average plastic strain $\bar{\gamma}^{\alpha}$

$$
\rho^{\alpha}(\boldsymbol{x})=\rho_{\text {sat }} \bar{\gamma}^{\alpha} \frac{b}{h} \sqrt{\frac{\left(1-2 \frac{x_{2}}{h}\right)^{2}}{\frac{x_{2}}{h}\left(1-\frac{x_{2}}{h}\right)}}+\rho_{0},
$$




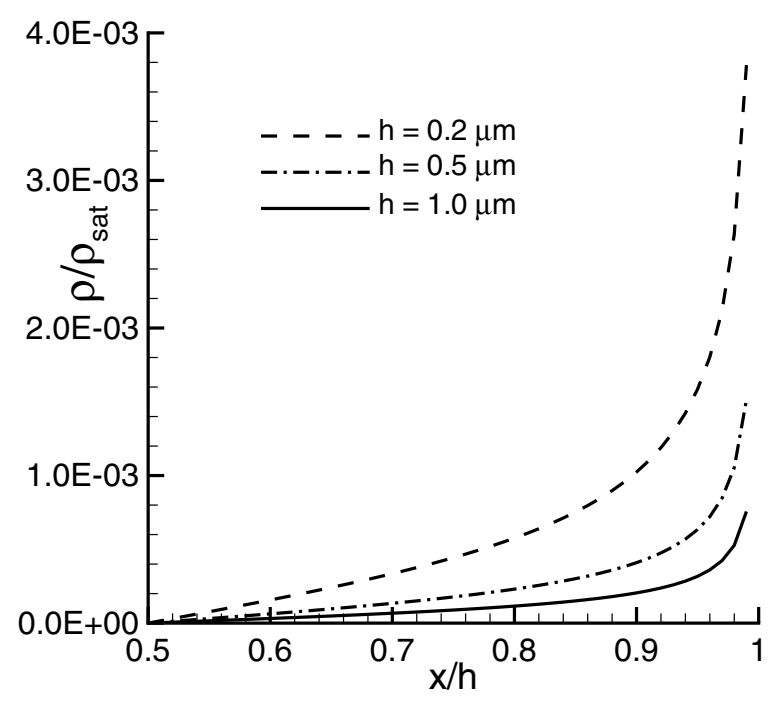

Figure 2. Average dislocation density profile across the film thickness

Table 1. Material properties from [30,31].

\begin{tabular}{llllll}
\hline Material & $v$ & $\alpha\left(\mathrm{K}^{-1}\right)$ & $C_{11}(\mathrm{GPa})$ & $C_{12}(\mathrm{GPa})$ & $C_{44}(\mathrm{GPa})$ \\
\hline $\mathrm{Cu}$ & 0.31 & $4.510^{-6}$ & 168.00 & 121.00 & 75.00 \\
\hline
\end{tabular}

where $\rho_{\text {sat }}=4 / \pi b^{2}$ and $\rho_{0}$ is an initial dislocation density set to $10^{11} \mathrm{~m}^{-2}$. Figure 2 shows the dislocation density profile along the film thickness for $\bar{\gamma}^{\alpha}=0.3$. The dislocation profile is symmetric with respect to the center of the film $x_{2} / h=0.5$ and the distance is normalized with respect to the film thickness. For the same average strain the dislocation density increases as the film thickness decreases to accommodate the same amount of plastic strain $\bar{\gamma}$ in a reduced domain. Equation (3.8) is the choice to describe the evolution of the dislocation density in the model described in section 2 for each slip system.

\section{Results}

The evolution of a thin film under thermal loading provides an example of the application of the model described in the previous sections. We simulate the mechanical response of a metallic thin film with a thickness ranging from 0.2 to $2.0 \mu \mathrm{m}$ exposed to thermal loading. A typical manufacturing process consists of several cycles where the temperature is raised and then cooled down to room temperature. In the present simulations the film is cooled down from 600 to $300 \mathrm{~K}$ to simulate the level of stresses at which thin films are subjected during fabrication of microelectronics [30].

We consider the material of the film to be copper single crystal within a rigid substrate and passivation. The single crystal is oriented such that the normal of the passivation and substrate interfaces are in the $(0,0,1)$ direction and the slip planes are in the $\{1,1,1\}$ family. Under the current loading conditions slip systems A6, B5, C5 and D6 are not active while the other slip systems are activated (here we adhere to the Schimd and Boas convention for fcc crystals). Material parameters of copper used in the simulations are listed in table 1. 


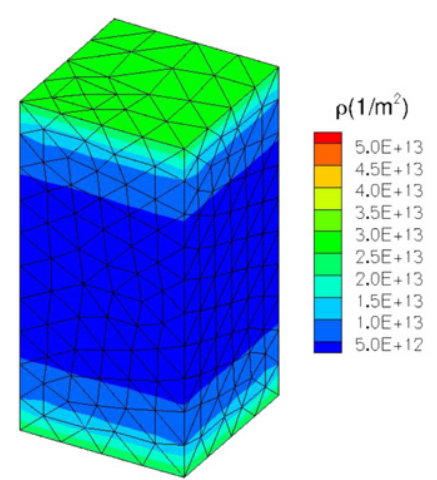

(a)

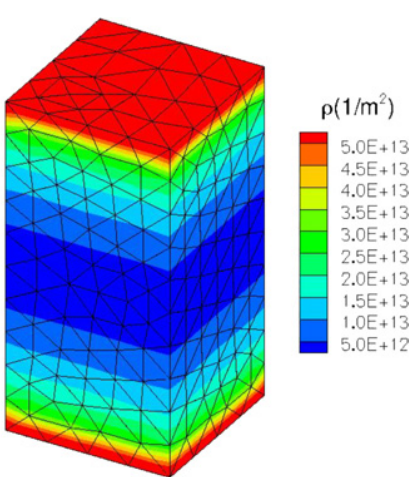

(b)

Figure 3. Simulated dislocation density in a $0.5 \mu \mathrm{m}$ thickness thin film with passivated interfaces. (a) $T=450 \mathrm{~K}$. (b) $T=300 \mathrm{~K}$.

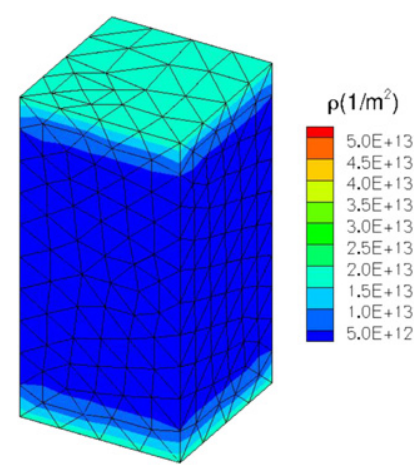

(a)

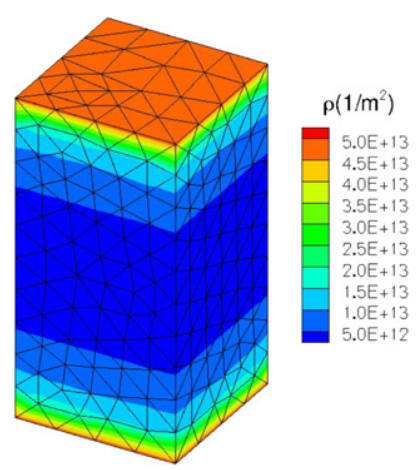

(b)

Figure 4. Simulated dislocation density in a $1 \mu \mathrm{m}$ thickness thin film with passivated interfaces. (a) $T=450 \mathrm{~K}$. (b) $T=300 \mathrm{~K}$

Figures 3 and 4 show the simulated dislocation density profile across the film thickness for $h=0.5 \mu \mathrm{m}$ and $h=1 \mu \mathrm{m}$, respectively. Figures 3(a) and 4(a) show the dislocation density at temperature $T=450 \mathrm{~K}$ and Figures $3(b)$ and $4(b)$ show the dislocation density at room temperature after cooling down from $600 \mathrm{~K}$. We start all simulations with a constant dislocation density $\rho_{0}=10^{11} \mathrm{~m}^{-2}$, and we assume $\rho_{\text {sat }}=1 \times 10^{19} \mathrm{~m}^{-2}$. As the temperature decreases, the stress raises generating plastic deformation. Therefore, the dislocation density increases and dislocations get trapped at the hard interfaces creating layers of high dislocation density at these interfaces. This layer of high dislocation density close to the interfaces has been observed in experiments and simulations $[12,15,16]$.

It can be noted in figures 3 and 4 that for the same temperature the average dislocation density is higher for the film with smaller thickness. This agrees with discrete and phase-field dislocation simulations that show that as the film thickness reduces, more dislocations are necessary to accommodate the same amount of plastic deformation [12,15]. This relation is clear in figure 5. This figure shows the evolution of the average dislocation density as a function of the average stress in the direction of the film thickness. As the film thickness is decreased to $0.2 \mu \mathrm{m}$ the average dislocation density almost duplicates its value. 


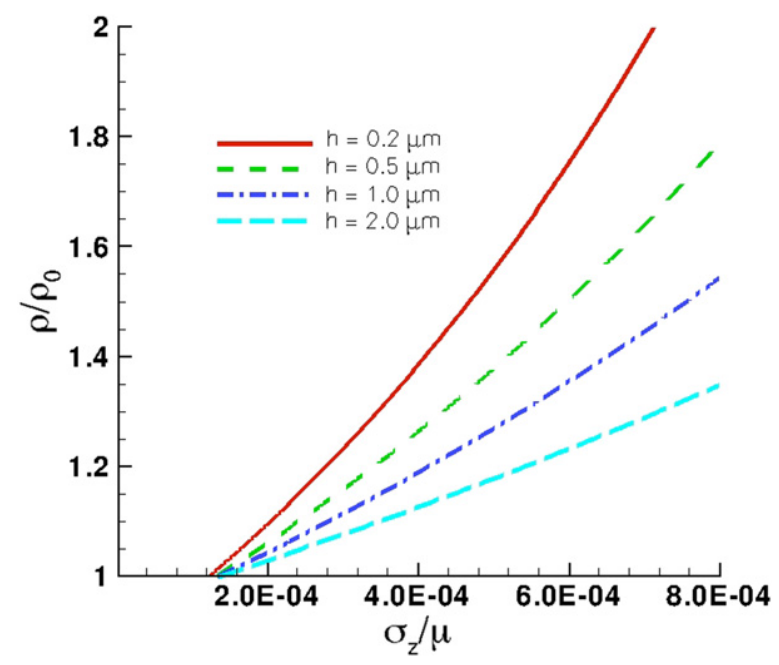

Figure 5. Simulated stress versus average dislocation density.

This rise in dislocation density as the thickness decreases is responsible for the dependence of hardening on the film thickness. Figure 6 shows the simulated stress strain curves; it is clear that the strain hardening increases as the film thickness is reduced in agreement with experiments $[6,4,17,18]$. It should be noticed that in the flow rule $(2.4)$ the yield stress does not depend explicitly on the film thickness. The dependence is given by the dislocation density and, as in all simulations, initially the dislocation density is set to be $\rho_{0}=10^{11} \mathrm{~m}^{-2}$ the initial yield stress is independent of the film thickness as can be observed in figure 6 . The dependence of the initial yield stress can be incorporated into the model if different initial dislocation densities are considered for different film thicknesses. From the stress strain curves in figure 6 we calculate the average stress at $0.2 \%$ plastic strain for different thicknesses. We find a relation of the form given by equation (1.1) with an exponent $n=0.19 \pm 0.05$. It bears emphasis that this value does not agree with dislocation dynamics simulations [15] because here we do not take into account the effect of film thickness on the yield stress.

To quantify the strain hardening dependence on the film thickness, we calculated the difference in stress for different strain values following Gruber et al [6]. Figure 7 shows the difference in stress at $0.4 \%$ and $0.2 \%, 0.6 \%$ and $0.2 \%$ and $0.8 \%$ and $0.2 \%$ plastic strain. Independent of the amount of plastic strain an increase in strain hardening is observed with decreasing film thickness in good agreement with experiments [6]. In our simulations we find an exponent $n_{d}=0.5 \pm 0.1$ in very good agreement with experimental observation.

\section{Summary and concluding remarks}

We carry finite elements simulations of the mechanical response of a single crystal thin film with stresses arising from thermal expansion. The film thickness in the simulations ranges from 0.2 to $2.0 \mu \mathrm{m}$. In the current model we incorporate the evolution of dislocation density directly from phase-field dislocation simulations. The dislocation density increases as the plastic deformation builds up forming a structure with higher dislocation density close to the interfaces between the crystal and substrate and passivation. 


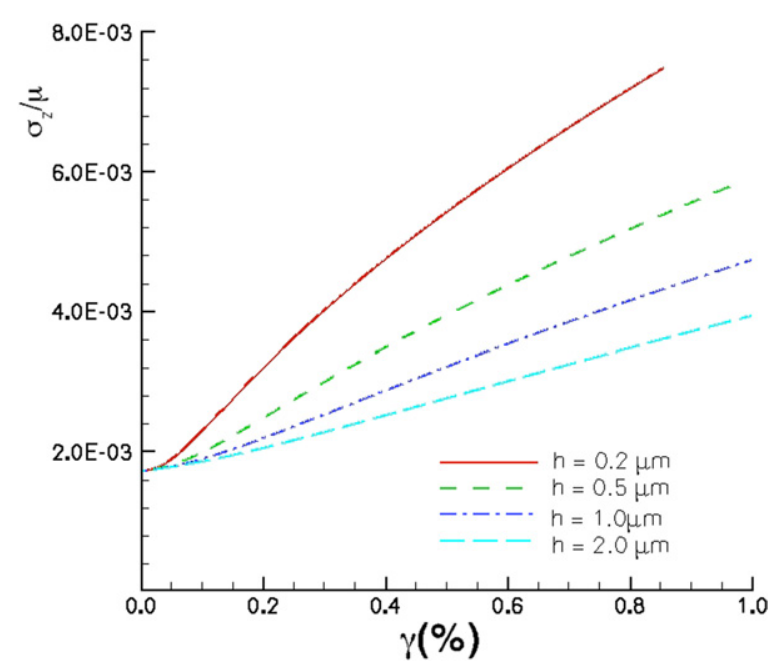

Figure 6. Simulated stress-plastic strain curves.

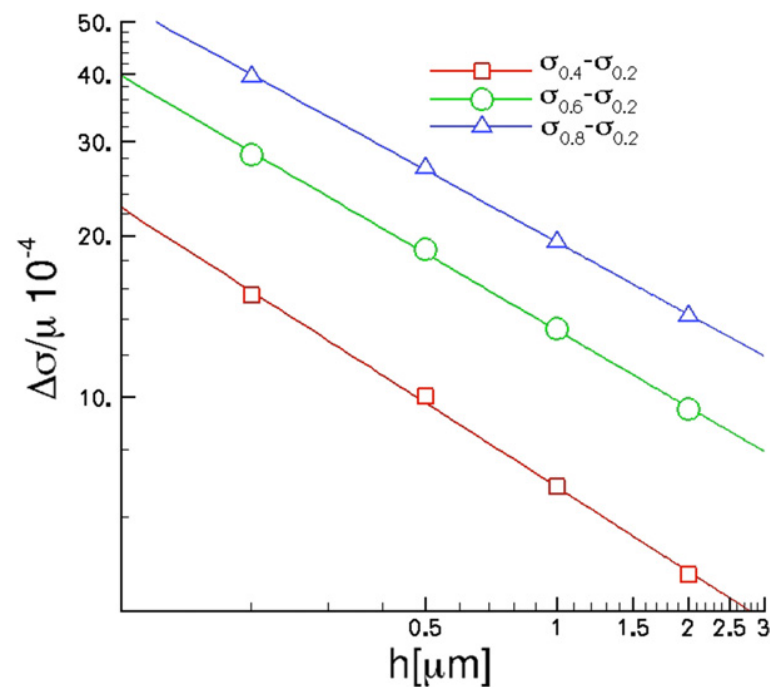

Figure 7. Strain hardening dependence on the film thickness. Different curves show the difference in stress for $0.4 \%$ and $0.2 \%, 0.6 \%$ and $0.2 \%$ and $0.8 \%$ and $0.2 \%$ plastic strain.

Phase-field dislocation simulations show that the yield stress increases as the film thickness decreases with an exponent $n=1$. This exponent corresponds to the onset of plastic deformation, in particular the stress needed to nucleate one dislocation in a domain with characteristic size $h$. In the current simulations this effect is not incorporated and $\tau_{y}$ in equation (2.4) is kept constant in all simulations as can be seen in figure 6.

Our finite element simulations capture the dependence of strain hardening on the film thickness in very good agreement with experimental observation [6]. As the film thickness decreases a higher dislocation density is required to accommodate the same amount of plastic strain. Also, dislocation density increases substantially close to the hard interfaces augmenting the strain hardening effect in thinner films. All these effects cause the strain hardening as the 
film thickness decreases following a power law with an exponent $n_{d}=0.5 \pm 0.1$ in very good agreement with experimental observation.

\section{Acknowledgments}

The authors are grateful for the support from the US Department of Energy, Office of Basic Energy Sciences under contract DEFG-02-07ER47398 and Department of Energy (National Nuclear Security Administration) under award number DE-FC52-08NA28617. They would like to thank Professor Alberto Cuitiño for sharing his single crystal plasticity finite elements algorithm with them.

\section{References}

[1] Hall E O 1951 The deformation and ageing of mild steel Proc. Phys. Soc. Lond. 64 747-53

[2] Petch N J 1953 Cleavage strength of polycrystals J. Iron Steel Inst. 174 25-28

[3] Espinosa H D, Prorok B C and Peng B 2004 Plasticity size effects in free-standing submicron polycrystalline fcc films subjected to pure tension J. Mech. Phys. Solids 52 667-89

[4] Xiang Y, Tsui T Y and Vlassak J J 2006 The mechanical properties of freestanding electroplated cu thin films J. Mater. Res. 21 1607-18

[5] Venkaltraman R and Bravman C 1992 Separation of film thickness and grain boundary strengthening in Al thin films on Si J. Mater. Res. 7 2040-8

[6] Gruber B A, Bohm J, Onuseit F, Wanner A, Spolenak R and Arzt E 2008 Size effects on yield strength and strain hardening fro ultra-thin cu films with and without passivation: a study by synchrotron and bulge test techniques Acta Metall. 56 2318-35

[7] Baker S P 2001 Plastic deformation and strength of materials in small dimensions Mater. Sci. Eng. A 319-321 16-23

[8] Hemker K J and Sharpe W N 2007 Microscale characterization of mechanical properties Annu. Rev. Mater. Sci. 37 93-126

[9] Matthews J W, Mader S and Light T B 1970 Accomodation of misfit across the interface between crystals of semiconducting element compounds J. Appl. Phys. 41 3800-4

[10] Freund L B 1990 A criterion for arrest of a threading dislocation in a strained epitaxial layer due to an interface misfit dislocation in its path J. Appl. Phys. 68 2073-80

[11] Nix W D 1998 Yielding and strain hardening of thin metal films on substrates Scr. Mater. 39 545-54

[12] Nicola L, Needleman A and Van der Giessen E 2003 Discrete dislocation analysis of size effects in thin films J. Appl. Phys. 93 5920-8

[13] Arsenlis A, Parks D M, Becker R and Bulatov V V 2004 On the evolution of crystallographic dislocation density in non-homogeneously deforming crystals J. Mech. Phys. Solids 52 1213-46

[14] Koslowski M 2007 Scaling laws in plastic deformation Phil. Mag. 87 1175-84

[15] Hunter A and Koslowski M 2008 Direct calculation of material parameters for gradient plasticity J. Mech. Phys Solids 56 3181-90

[16] Minor A M, Lilleoden E T, Stach E A and Morris J W Jr 2004 Direct observation of incipient plasticity during indentation of Al J. Mater. Res. 19 176-82

[17] Wang L and Prorok B C 2008 Characterization of the strain rate dependent behavior of nanocrystalline gold films J. Mater. Res. 23 55-65

[18] Chasiotis I, Bateson C, Timpano K, McCarthy A S, Barker N S and Stanec J R 2007 Strain rate effects on the mechanical behavior of nanocrystalline Au films Thin Solid Films 515 3183-9

[19] Asaro R J 1983 Micromechanics of crystals and polycrystals Adv. Appl. Mech. 23 1-115

[20] Cuitiño A M and Ortiz M 1993 Computational modeling of single crystals Modelling Simul. Mater. Sci. Eng. $1255-63$

[21] Nix W D and Gao H 1997 Indentation size effects in crystalline materials: A law for strain gradient plasticity J. Mech. Phys. Solids $\mathbf{4 6} 422-5$

[22] Fleck N A and Hutchinson J W 1997 Strain gradient plasticity Adv. Appl. Mech. 33 296-358

[23] Stolken J S and Evans A G 1998 Microbend test method for measuring the plasticity length scale Acta Mater. 46 5109-15

[24] Acharya A and Bassani J L 2000 Lattice incompatibility and a gradient theory of crystal plasticity J. Mech. Phys. Solids 48 1565-95 
[25] Gurtin M E 2002 A gradient theory of single-crystal viscoplasticity that accounts for geometrically necessary dislocations J. Mech. Phys. Solids 50 5-32

[26] Franciosi P and Zaoui A 1982 Multislip in f.c.c. crystals: A theoretical approach compared with experimental data Acta Metall. 301627

[27] Koslowski M, Cuitiño A and Ortiz M 2002 A phase-field theory of dislocations dynamics, strain hardening and hysteresis in ductile single crystals J. Mech. Phys. Solids 50 2597-635

[28] Garroni A and Muller S 2006 A variational model for dislocations in the line tension limit Arch. Ration. Mech. Anal. 181 535-78

[29] Focardo M and Garroni A 2007 A 1D macroscopic phase field model for dislocations and second order $\gamma$-limit Multiscale Modeling Simul. 6 1098-124

[30] Lee J and Sauter Mack A 1998 Finite element simulation of a stress history during the manufactering process of thin film stacks in VLSI structures IEEE Trans. Semicond. Manuf. 11 458-64

[31] Hirth J P and Lothe J 1968 Theory of Dislocations (New York: McGraw-Hill) 